Manuscript received 04.05.2020

Accepted: 28.06 .2020

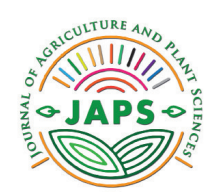

In print: ISSN 2545-4447

On line: ISSN 2545-4455

doi: https://doi.org/10.46763/JAPS2010041r

Original scientific paper

\title{
INVESTIGATION OF THE CORRELATIONS BETWEEN QUANTITATIVE TRAITS WHICH DETERMINE YIELD IN THE VINE CULTIVAR BOLGAR AND THE HYBRID COMBINATION BOLGAR X RUSSALKA 1
}

\author{
Venelin Roychev ${ }^{1^{*}}$, Dushko Nedelkovski ${ }^{2}$, Klime Beleski ${ }^{2}$ \\ ${ }^{1}$ Agricultural University - Plovdiv, R. Bulgaria \\ ${ }^{2}$ Ss Cyril and Methodius University, Institute of Agriculture - Skopje, Republic of North Macedonia \\ *Corresponding author: roytchev@yahoo.com
}

\begin{abstract}
The correlations between quantitative traits, which determine yield in the vine cultivar Bolgar and $F_{1}$ progeny of the hybrid combination Bolgar x Russalka 1 have been investigated through Path analysis. It has been found that there are no highly significant traits for the formation of yield from this cultivar, for which the correlation coefficients, direct and total indirect influences have positive values. Positive correlations have been reported between the two parent cultivars and F1 progeny for the traits: shoot and fruiting shoot fertility coefficient, cluster width, total number of shoots, fruiting shoots and clusters. All studied fertility coefficients in the cultivar Bolgar exert positive direct influences, determined by moderate correlations, on the seedlings from F1 progeny. The correlations of the other traits and the influence of separate parent cultivars have positive or negative values, which can be used in the selection of valuable elite forms.
\end{abstract}

Key words: quantitative traits, yield; seeded and seedless vine cultivar; F1 progeny; correlations; direct and indirect influences; Path analysis

\section{INTRODUCTION}

It is known that, by means of Path analysis it is possible to determine the degree, to which the variability of a certain quantitative trait influences the change of a single trait or a group of other traits. The total correlation and the relative participation of each trait in yield formation are established through this method, and it allows their comparing with results from other experiments. The advantages of Path analysis combined with the analytical potential of the production function are a model, which can be efficiently applied in the experimental procedures in viticulture (Mokreva 2004, 2007). The type of the effect from the influence of a certain significant agrobiological factor, combined with the remaining ones, on the phenotypic manifestation of the variable value - the cumulative trait (yield), is also of interest. Their application is especially important and valuable in the complicated development of new table seeded and seedless vine cultivars (Smirnov 1977; Golodriga et al., 1985; Pospisilova, Palenik 1988; Valchev 1990; Troshin 1990, 1997). The purpose of the current investigation is to determine the degree of correlations between quantitative traits and their relative participation in yield formation for the vine cultivar Bolgar and $F_{1}$ progeny of the hybrid combination Bolgar x Russalka 1.

\section{MATERIAL AND METHODS}

During seven consecutive years, in 30 plants from $F_{1}$ progeny of the hybrid combination Bolgar $\left(\mathrm{P}_{1}\right.$ - seeded) $x$ Russalka 1 ( $\mathrm{P}_{2}$ - seedless), 21 quantitative traits were determined, related to the phenology, fertility, quality and grape yield
(Bulgarian Ampelography, 1990; Roychev 2012). The experimental results were processed by means of Path analysis (Rokitskii 1973; Lidanski 1988). The studied traits were conditionally divided into six groups. The direct and indirect 
influences of the indicated traits of parent cultivars on yield formation in seedlings and the degree of correlations between them were analyzed. The presented results are a part of a larger-scale research related to the application of Path analysis in selection for the obtaining of new seeded and seedless vine cultivars.

\section{RESULTS AND DISCUSSION}

In the cultivar Bolgar $\left(P_{1}\right)$ few traits from different groups manifest high, significant and moderate correlations with yield (Table 1). Most often, the high direct positive effect is eliminated by almost the same in size total indirect influence, deriving from the interdependence of the other traits or vice versa. This is typical not only for the traits with higher correlation coefficients $(0,625)$ - total number of clusters, $(0,530)$ - total number of fruiting shoots, $(0,476)$ - total number of shoots, $(0,357)$ - berry length, $(0,383)$ - berry width, $(0,357)$ shoot fertility coefficient, $(0,288)$ - main shoot fertility coefficient, but also for all remaining ones. There are no traits significant for the yield, in which the correlation coefficients, direct and total indirect influences have positive values, which means that the yield in this cultivar depends on numerous different factors and their interaction.

Table 1.

The traits which exert positive influence on yield formation in the cultivar Bolgar (P1) are in the first, second, third and sixth group, with total relative participation $95,6 \%$ (Table 2). The most significant presence belongs to all traits connected with the actual fertility of vines
$(59,2 \%)$. Out of them, the larger share belongs to total number of clusters (24,8\%) and fruiting shoots $(17,9 \%)$, as well as the three fertility coefficients - totally $(16,5 \%)$. Comparatively more traits from a larger number of groups participate in yield formation for this cultivar.

Table 2. Relative participation of traits in the formation of grape yield from the seeded cultivar Bolgar $\left(\mathrm{P}_{1}\right)$.

\begin{tabular}{|c|c|c|c|}
\hline \multirow[b]{2}{*}{ Groups } & \multirow[b]{2}{*}{ № } & Total yield variation & 100,0 \\
\hline & & $\begin{array}{l}\text { Total relative participation of the most important traits } \\
\qquad 95,6 \% \text {, from which: }\end{array}$ & $\%$ \\
\hline \multirow{3}{*}{ I } & $x_{1}$ & Shoot fertility coefficient & 8.1 \\
\hline & $x_{2}$ & Main shoot fertility coefficient & 5.3 \\
\hline & $x_{3}$ & Fruiting shoot fertility coefficient & 3.1 \\
\hline II & $x_{6}$ & Cluster length $(\mathrm{cm})$ & 2.5 \\
\hline \multirow{2}{*}{ III } & $x_{9}$ & Berry length (mm) & 8.1 \\
\hline & $x_{10}$ & Berry width (mm) & 9.3 \\
\hline \multirow{4}{*}{$\mathrm{VI}$} & $\mathrm{x}_{18}$ & Total number of buds & 2.1 \\
\hline & $\mathrm{X}_{19}$ & Total number of shoots & 14.4 \\
\hline & $x_{20}$ & Total number of fruiting shoots & 17.9 \\
\hline & $x_{21}$ & Total number of clusters & 24.8 \\
\hline & & Other traits & 4.4 \\
\hline
\end{tabular}




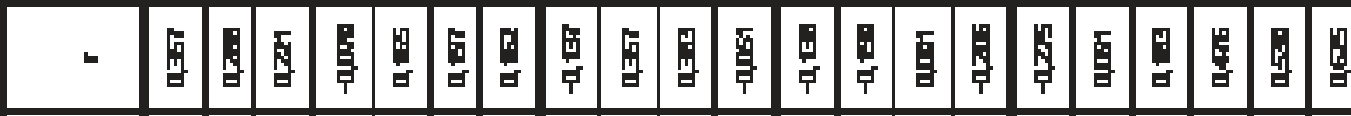

1)

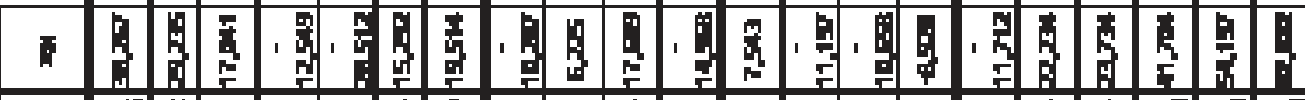

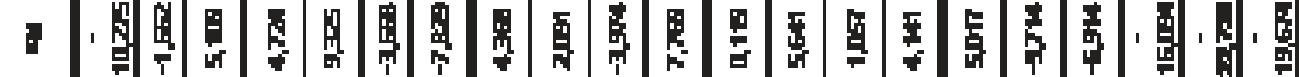

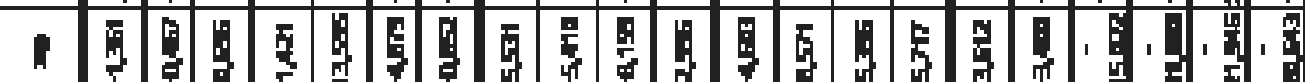

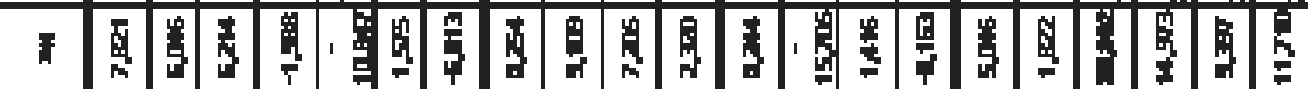

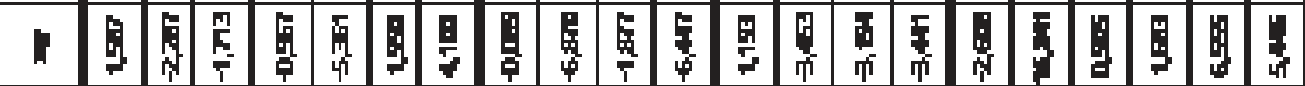

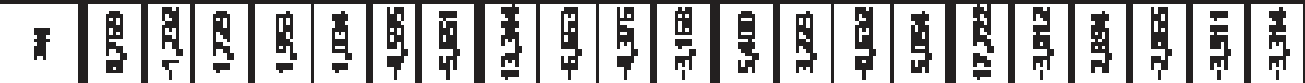

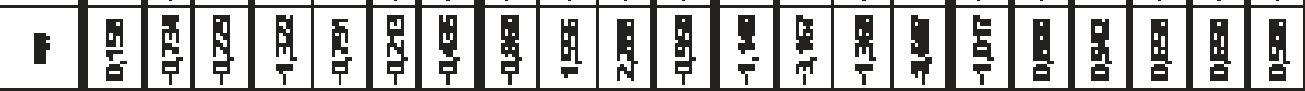

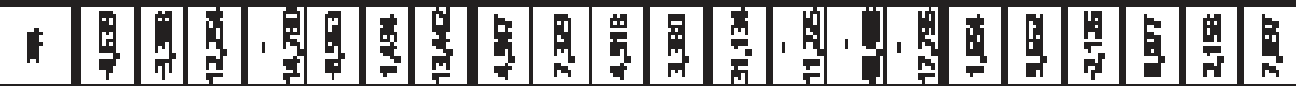

180.000

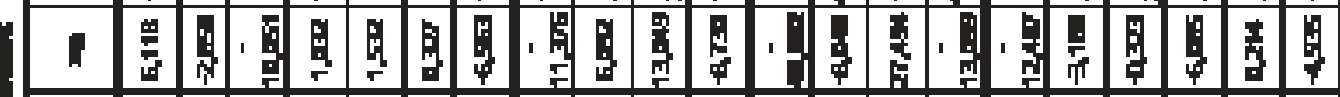

$3 \mathrm{H}$

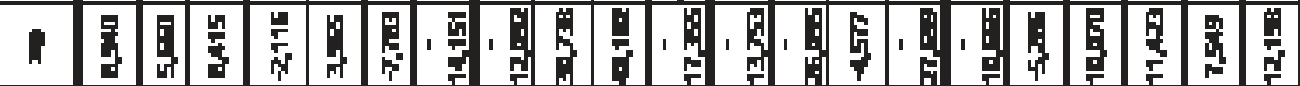

하

- 5

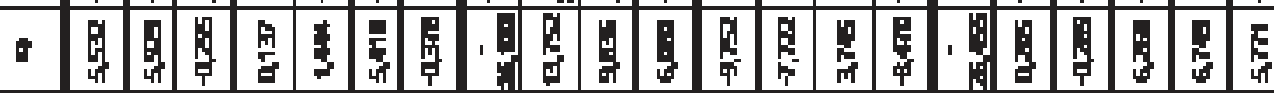

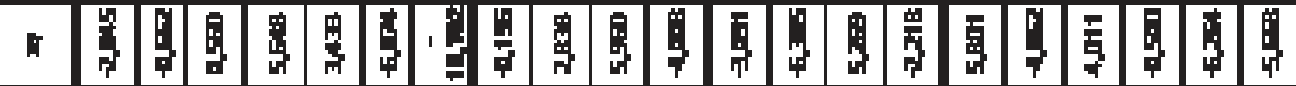

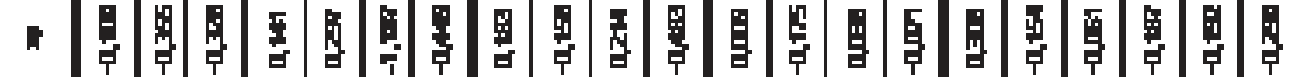

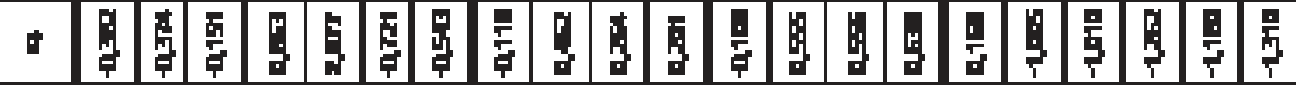

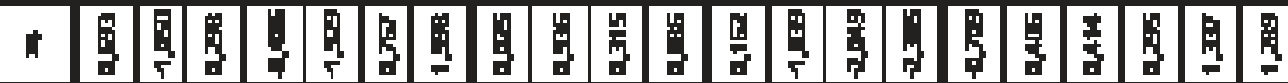

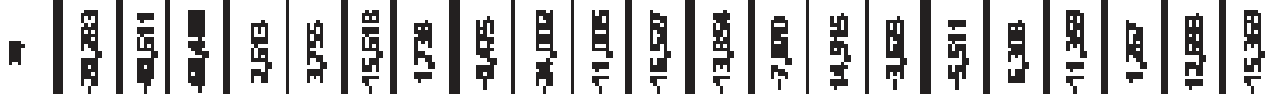

-

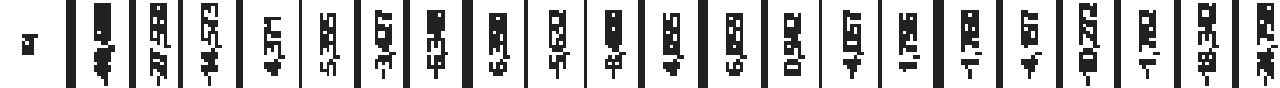

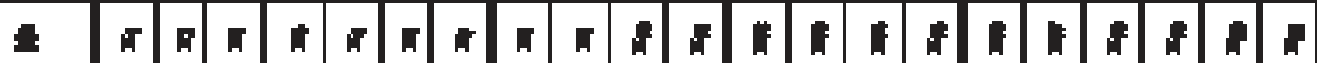

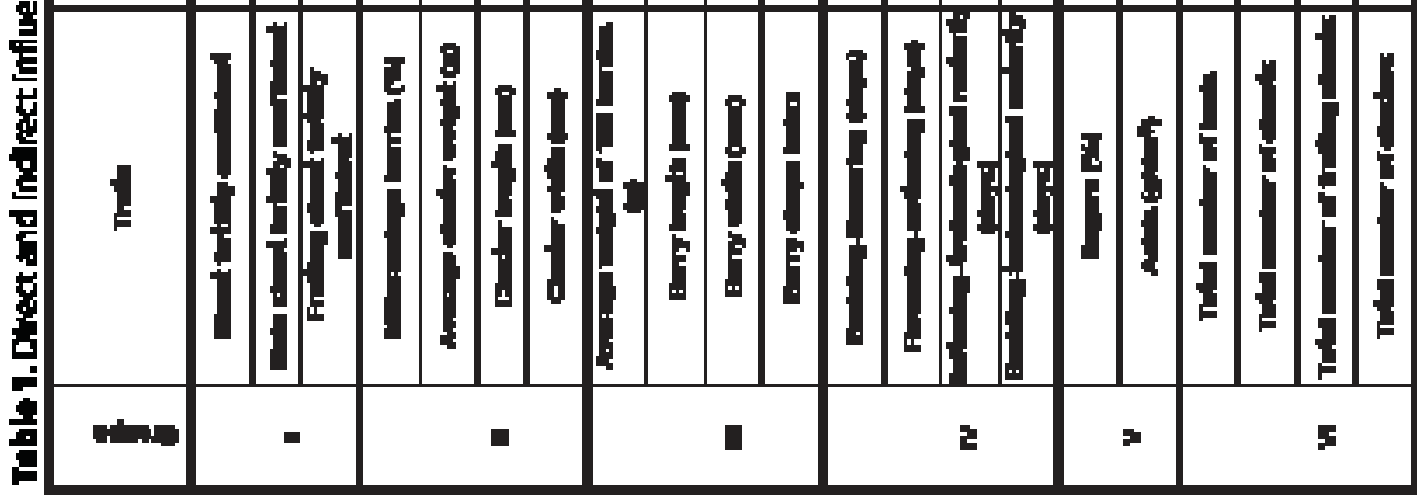


The structure of correlations between separate parents and $F_{1}$ progeny by groups of traits is different (Table 3). In the first group, the correlations are positive at shoot fertility coefficient $(0,176)-P_{1},(0,235)-P_{2}$ and fruiting shoot fertility coefficient $(0,165)-P_{1},(0,382)-P_{2}$; in the second group - cluster width $(0,138)-P_{1^{\prime}}$ $(0,608)-P_{2}$, in the sixth group - total number of shoots $(0,305)-P_{1},(0,009)-P_{2}$, total number of fruiting shoots $(0,155)-P_{1},(0,057)-P_{2}$ and total number of clusters $(0,291)-\mathrm{P}_{1},(0,027)-\mathrm{P}_{2}$. In all traits in the fourth group - phenophases and periods, fifth group - chemical composition, and sixth group - actual fertility of vine, the positive correlations prevail of the cultivar Bolgar $\left(\mathrm{P}_{1}\right)$ with $F_{1}$ progeny, and in the first group - of the cultivar Russalka $1\left(\mathrm{P}_{2}\right)$. Except for berry shape index for Bolgar $\left(P_{1}\right)$, both parent cultivars have negative correlations for the berry traits. We should also point out the higher positive correlations of Bolgar $\left(P_{1}\right)$ with $F_{1}$ progeny regarding the traits total number of buds $(0,385)$, acids $(0,578)$, budding-flowering $(0,399)$ and cluster length $(0,525)$, and of Russalka $1\left(P_{2}\right)$ - main shoot fertility coefficient $(0,313)$, millerandage berries $(0,358)$ and average cluster weight $(0,278)$.

Table 3. Correlation coefficients between the traits of the studied vine cultivars Bolgar $\left(P_{1}\right)$, Russalka $1\left(P_{2}\right)$ and the plants from the hybrid combination $-F_{1}$ progeny.

\begin{tabular}{|c|c|c|c|c|c|c|c|}
\hline Groups & № & Traits & \multicolumn{2}{|c|}{ Cultivars } & \multirow{2}{*}{$\begin{array}{c}\mathrm{F}_{1} \\
1 \\
\end{array}$} & \multirow{2}{*}{$\begin{array}{c}P_{1} \\
0,176 \\
\end{array}$} & \multirow{2}{*}{$\begin{array}{c}P_{2} \\
0,235\end{array}$} \\
\hline \multirow{9}{*}{ I } & \multirow{3}{*}{1} & \multirow{3}{*}{$\begin{array}{l}\text { Shoot fertility } \\
\text { coefficient }\end{array}$} & $F_{1}$ progeny & $\mathrm{F}_{1}$ & & & \\
\hline & & & Bolgar & $P_{1}$ & & 1 & $-0,133$ \\
\hline & & & Ryssalka 1 & $P_{2}$ & & & 1 \\
\hline & \multirow{6}{*}{3} & \multirow{3}{*}{$\begin{array}{l}\text { Main shoot fertility } \\
\text { coefficient }\end{array}$} & $F_{1}$ progeny & $\mathrm{F}_{1}$ & 1 & $-0,098$ & 0,313 \\
\hline & & & Bolgar & $P_{1}$ & & 1 & 0,124 \\
\hline & & & Ryssalka 1 & $\mathrm{P}_{2}$ & & & 1 \\
\hline & & \multirow{3}{*}{$\begin{array}{l}\text { Fruiting shoot fertility } \\
\text { coefficient }\end{array}$} & $F_{1}$ progeny & $\mathrm{F}_{1}$ & 1 & 0,165 & 0,382 \\
\hline & & & Bolgar & $\mathrm{P}_{1}$ & & 1 & $-0,236$ \\
\hline & & & Ryssalka 1 & $\mathrm{P}_{2}$ & & & 1 \\
\hline \multirow{12}{*}{ II } & \multirow{3}{*}{4} & \multirow{3}{*}{$\begin{array}{l}\text { Millerandage berries } \\
(\%)\end{array}$} & $\mathrm{F}_{1}$ progeny & $\mathrm{F}_{1}$ & 1 & $-0,042$ & 0,358 \\
\hline & & & Bolgar & $P_{1}$ & & 1 & 0,218 \\
\hline & & & Ryssalka 1 & $\mathrm{P}_{2}$ & & & 1 \\
\hline & & & $F_{1}$ progeny & $\mathrm{F}_{1}$ & 1 & $-0,112$ & 0,278 \\
\hline & 5 & Average cluster weight & Bolgar & $P_{1}$ & & 1 & 0,135 \\
\hline & & & Ryssalka 1 & $\mathrm{P}_{2}$ & & & 1 \\
\hline & & & $F_{1}$ progeny & $\mathrm{F}_{1}$ & 1 & 0,525 & $-0,350$ \\
\hline & 6 & Cluster length $(\mathrm{cm})$ & Bolgar & $\mathrm{P}_{1}$ & & 1 & $-0,270$ \\
\hline & & & Ryssalka 1 & $P_{2}$ & & & 1 \\
\hline & & & $F_{1}$ progeny & $\mathrm{F}_{1}$ & 1 & 0,138 & 0,608 \\
\hline & 7 & Cluster width $(\mathrm{cm})$ & Bolgar & $P_{1}$ & & 1 & 0,117 \\
\hline & & & Ryssalka 1 & $\mathrm{P}_{2}$ & & & 1 \\
\hline & & & $F_{1}$ progeny & $\mathrm{F}_{1}$ & 1 & $-0,294$ & $-0,190$ \\
\hline & 8 & Average weight of 100 & Bolgar & $P_{1}$ & & 1 & $-0,044$ \\
\hline & & berries $(\mathrm{g})$ & Ryssalka 1 & $P_{2}$ & & & 1 \\
\hline & & & $F_{1}$ progeny & $\mathrm{F}_{1}$ & 1 & $-0,048$ & $-0,251$ \\
\hline III & 9 & Berry length (mm) & Bolgar & $P_{1}$ & & 1 & 0,174 \\
\hline & & & Ryssalka 1 & $\mathrm{P}_{2}$ & & & 1 \\
\hline & & & $\mathrm{F}_{1}$ progeny & $\mathrm{F}_{1}$ & 1 & $-0,016$ & $-0,075$ \\
\hline & 10 & Berry width (mm) & Bolgar & $P_{1}$ & & 1 & $-0,327$ \\
\hline & & & Ryssalka 1 & $\mathrm{P}_{2}$ & & & 1 \\
\hline & & & $F_{1}$ progeny & $\mathrm{F}_{1}$ & 1 & 0,035 & $-0,294$ \\
\hline & 11 & Berry shape index & \begin{tabular}{|l|} 
Bolgar \\
\end{tabular} & $P_{1}$ & & 1 & 0,295 \\
\hline & & & Ryssalka 1 & $P_{2}$ & & & 1 \\
\hline
\end{tabular}




\begin{tabular}{|c|c|c|c|c|c|c|c|}
\hline \multirow{12}{*}{ IV } & \multirow{3}{*}{12} & \multirow{3}{*}{$\begin{array}{l}\text { Budding-flowering } \\
\text { (days) }\end{array}$} & $\mathrm{F}_{1}$ progeny & $\mathrm{F}_{1}$ & 1 & 0.399 & -0.106 \\
\hline & & & Bolgar & $P_{1}$ & & 1 & -0.220 \\
\hline & & & Ryssalka 1 & $\mathrm{P}_{2}$ & & & 1 \\
\hline & \multirow{3}{*}{13} & \multirow{3}{*}{$\begin{array}{l}\text { Flowering-softening } \\
\text { (days) }\end{array}$} & $F_{1}$ progeny & $\mathrm{F}_{1}$ & 1 & 0.088 & -0.042 \\
\hline & & & Bolgar & $P_{1}$ & & 1 & 0.317 \\
\hline & & & Ryssalka 1 & $\mathrm{P}_{2}$ & & & 1 \\
\hline & \multirow{3}{*}{14} & \multirow{3}{*}{$\begin{array}{l}\text { Softening- } \\
\text { technological maturity } \\
\text { (days) }\end{array}$} & $F_{1}$ progeny & $F_{1}$ & 1 & 0.089 & -0.440 \\
\hline & & & Bolgar & $\mathrm{P}_{1}$ & & 1 & 0.255 \\
\hline & & & Ryssalka 1 & $\mathrm{P}_{2}$ & & & 1 \\
\hline & \multirow{3}{*}{15} & \multirow{3}{*}{$\begin{array}{l}\text { Budding-technological } \\
\text { maturity (days) }\end{array}$} & $F_{1}$ progeny & $F_{1}$ & 1 & 0.135 & -0.228 \\
\hline & & & Bolgar & $P_{1}$ & & 1 & 0.305 \\
\hline & & & Ryssalka 1 & $\mathrm{P}_{2}$ & & & 1 \\
\hline \multirow{6}{*}{ V } & \multirow{3}{*}{16} & \multirow{3}{*}{ Sugars (\%) } & $F_{1}$ progeny & $\mathrm{F}_{1}$ & 1 & 0.169 & -0.208 \\
\hline & & & Bolgar & $P_{1}$ & & 1 & 0.186 \\
\hline & & & Ryssalka 1 & $\mathrm{P}_{2}$ & & & 1 \\
\hline & \multirow{3}{*}{17} & \multirow{3}{*}{ Acids $\left(\mathrm{g} / \mathrm{dm}^{3}\right)$} & $\mathrm{F}_{1}$ progeny & $\mathrm{F}_{1}$ & 1 & 0.578 & -0.019 \\
\hline & & & Bolgar & $P_{1}$ & & 1 & 0.159 \\
\hline & & & Ryssalka 1 & $\mathrm{P}_{2}$ & & & 1 \\
\hline \multirow{12}{*}{ VI } & \multirow{3}{*}{18} & \multirow{3}{*}{ Total number of buds } & $F_{1}$ progeny & $\mathrm{F}_{1}$ & 1 & 0.385 & -0.096 \\
\hline & & & Bolgar & $P_{1}$ & & 1 & 0.099 \\
\hline & & & Ryssalka 1 & $\mathrm{P}_{2}$ & & & 1 \\
\hline & \multirow{3}{*}{19} & \multirow{3}{*}{ Total number of shoots } & $F_{1}$ progeny & $\mathrm{F}_{1}$ & 1 & 0.305 & 0.009 \\
\hline & & & Bolgar & $\mathrm{P}_{1}$ & & 1 & 0.260 \\
\hline & & & Ryssalka 1 & $\mathrm{P}_{2}$ & & & 1 \\
\hline & \multirow{3}{*}{20} & \multirow{3}{*}{$\begin{array}{l}\text { Total number of fruiting } \\
\text { shoots }\end{array}$} & $F_{1}$ progeny & $\mathrm{F}_{1}$ & 1 & 0.155 & 0.057 \\
\hline & & & Bolgar & $P_{1}$ & & 1 & 0.065 \\
\hline & & & Ryssalka 1 & $\mathrm{P}_{2}$ & & & 1 \\
\hline & \multirow{3}{*}{21} & \multirow{3}{*}{ Total number of clusters } & $F_{1}$ progeny & $\mathrm{F}_{1}$ & 1 & 0.291 & 0.027 \\
\hline & & & Bolgar & $\mathrm{P}_{1}$ & & 1 & 0.053 \\
\hline & & & Ryssalka 1 & $\mathrm{P}_{2}$ & & & 1 \\
\hline
\end{tabular}

All fertility coefficients in the cultivar Bolgar $\left(\mathrm{P}_{1}\right)$ exert positive direct influence determined by a moderate correlation, on the seedlings from $F_{1}$ progeny - shoot fertility coefficient $(0,267 ; 0,235)$, main shoot fertility coefficient $(0,328 ; 0,313)$, fruiting shoot fertility coefficient $(0,433 ; 0,382)$ (Table 4$)$. Both parent cultivars have direct positive influence with a significant correlation coefficient on the trait millerandage berries. The direct influences of all traits in the sixth group of the cultivar Russalka $1\left(P_{2}\right)$ are positive, and of Bolgar $\left(P_{1}\right)$ - only in total number of fruiting shoots and clusters. Positive influences and correlations in the two cultivars were reported for cluster width, and in average cluster weight only their direct effects are positive. In sugars $(0,227)$, acids $(0,255)$, the phenological traits (except for buddingflowering), berry shape index $(0,306)$ and berry length $(0,162)$, the direct influence of Russalka 1 $\left(P_{2}\right)$ is positive, and of Bolgar $\left(P_{1}\right)$ - negative. In cluster length all influences and correlations are negative in both parent cultivars. 
Table 4. Direct and indirect influences of the studied parent vine cultivars Bolgar $\left(P_{1}\right)$ and Russalka $1\left(P_{2}\right)$ on the plants from the hybrid combination - $F_{1}$ progeny.

\begin{tabular}{|c|c|c|c|c|c|c|c|}
\hline Groups & № & Traits & \multicolumn{2}{|c|}{ Cultivars } & \multicolumn{2}{|c|}{$\begin{array}{l}\text { Direct and indirect } \\
\text { influences }\end{array}$} & \multirow{2}{*}{$\begin{array}{c}r \\
0,235\end{array}$} \\
\hline \multirow{6}{*}{ I } & \multirow[t]{2}{*}{1} & \multirow{2}{*}{$\begin{array}{l}\text { Shoot fertility } \\
\text { coefficient }\end{array}$} & Bolgar & $\mathrm{P}_{1}$ & 0,267 & $-0,032$ & \\
\hline & & & Ryssalka 1 & $\mathrm{P}_{2}$ & 0,047 & $-0,180$ & $-0,133$ \\
\hline & \multirow[t]{2}{*}{2} & \multirow{2}{*}{$\begin{array}{l}\text { Main shoot fertility } \\
\text { coefficient }\end{array}$} & Bolgar & $\mathrm{P}_{1}$ & 0,328 & $-0,015$ & 0,313 \\
\hline & & & Ryssalka 1 & $\mathrm{P}_{2}$ & $-0,032$ & 0,156 & 0,124 \\
\hline & \multirow[t]{2}{*}{3} & \multirow{2}{*}{$\begin{array}{l}\text { Fruiting shoot fertility } \\
\text { coefficient }\end{array}$} & Bolgar & $\mathrm{P}_{1}$ & 0,433 & $-0,051$ & 0,382 \\
\hline & & & Ryssalka 1 & $\mathrm{P}_{2}$ & 0,071 & $-0,307$ & $-0,236$ \\
\hline \multirow{8}{*}{ II } & \multirow[t]{2}{*}{4} & \multirow{2}{*}{$\begin{array}{l}\text { Millerandage berries } \\
(\%)\end{array}$} & Bolgar & $\mathrm{P}_{1}$ & 0,368 & $-0,010$ & 0,358 \\
\hline & & & Ryssalka 1 & $\mathrm{P}_{2}$ & $-0,015$ & 0,233 & 0,218 \\
\hline & \multirow[t]{2}{*}{5} & \multirow{2}{*}{$\begin{array}{l}\text { Average cluster } \\
\text { weight }(\mathrm{g})\end{array}$} & Bolgar & $\mathrm{P}_{1}$ & 0,297 & $-0,019$ & 0,278 \\
\hline & & & Ryssalka 1 & $\mathrm{P}_{2}$ & $-0,033$ & 0,168 & 0,135 \\
\hline & \multirow[t]{2}{*}{6} & \multirow[t]{2}{*}{ Cluster length $(\mathrm{cm})$} & Bolgar & $\mathrm{P}_{1}$ & $-0,288$ & $-0,062$ & $-0,350$ \\
\hline & & & Ryssalka 1 & $\mathrm{P}_{2}$ & $-0,151$ & $-0,119$ & $-0,270$ \\
\hline & \multirow[t]{2}{*}{7} & \multirow[t]{2}{*}{ Cluster width $(\mathrm{cm})$} & Bolgar & $\mathrm{P}_{1}$ & 0,603 & 0,005 & 0,608 \\
\hline & & & Ryssalka 1 & $\mathrm{P}_{2}$ & 0,083 & 0,034 & 0,117 \\
\hline \multirow{8}{*}{ III } & \multirow[t]{2}{*}{8} & \multirow{2}{*}{$\begin{array}{l}\text { Average weight of } \\
100 \text { berries }(\mathrm{g})\end{array}$} & Bolgar & $\mathrm{P}_{1}$ & $-0,222$ & 0,032 & $-0,190$ \\
\hline & & & Ryssalka 1 & $\mathrm{P}_{2}$ & 0,065 & $-0,109$ & $-0,044$ \\
\hline & \multirow[t]{2}{*}{9} & \multirow[t]{2}{*}{ Berry length (mm) } & Bolgar & $\mathrm{P}_{1}$ & $-0,243$ & $-0,008$ & $-0,251$ \\
\hline & & & Ryssalka 1 & $\mathrm{P}_{2}$ & 0,012 & 0,162 & 0,174 \\
\hline & 10 & Berry width $(\mathrm{mm})$ & Bolgar & $\mathrm{P}_{1}$ & $-0,080$ & 0,005 & $-0,075$ \\
\hline & & & Ryssalka 1 & $\mathrm{P}_{2}$ & 0,001 & $-0,328$ & $-0,327$ \\
\hline & 11 & Berry shape index & Bolgar & $\mathrm{P}_{1}$ & $-0,305$ & 0,011 & $-0,294$ \\
\hline & & & Ryssalka 1 & $\mathrm{P}_{2}$ & $-0,011$ & 0,306 & 0,295 \\
\hline & 12 & Budding-flowering & Bolgar & $\mathrm{P}_{1}$ & $-0,021$ & $-0,085$ & $-0,106$ \\
\hline & & (days) & Ryssalka 1 & $\mathrm{P}_{2}$ & $-0,008$ & $-0,212$ & $-0,220$ \\
\hline & 13 & Flowering-softening & Bolgar & $\mathrm{P}_{1}$ & $-0,070$ & 0,028 & $-0,042$ \\
\hline & & (days) & Ryssalka 1 & $\mathrm{P}_{2}$ & $-0,006$ & 0,323 & 0,317 \\
\hline IV & 14 & Softening- & Bolgar & $\mathrm{P}_{1}$ & $-0,467$ & 0,027 & $-0,440$ \\
\hline & & $\begin{array}{l}\text { technological } \\
\text { maturity (days)) }\end{array}$ & Ryssalka 1 & $\mathrm{P}_{2}$ & $-0,042$ & 0,297 & 0,255 \\
\hline & 15 & Budding- & Bolgar & $\mathrm{P}_{1}$ & $-0,274$ & 0,046 & $-0,228$ \\
\hline & & $\begin{array}{l}\text { technological } \\
\text { maturity (days) }\end{array}$ & Ryssalka 1 & $\mathrm{P}_{2}$ & $-0,037$ & 0,342 & 0,305 \\
\hline & 16 & Sugars (\%) & Bolgar & $\mathrm{P}_{1}$ & $-0,246$ & 0,038 & $-0,208$ \\
\hline V & & & Ryssalka 1 & $\mathrm{P}_{2}$ & $-0,041$ & 0,227 & 0,186 \\
\hline & 17 & Acids $\left(\mathrm{g} / \mathrm{dm}^{3}\right)$ & Bolgar & $\mathrm{P}_{1}$ & $-0,166$ & 0,147 & $-0,019$ \\
\hline & & & Ryssalka 1 & $\mathrm{P}_{2}$ & $-0,096$ & 0,255 & 0,159 \\
\hline & 18 & Total number of & Bolgar & $\mathrm{P}_{1}$ & $-0,158$ & 0,062 & $-0,096$ \\
\hline & & shoots & Ryssalka 1 & $\mathrm{P}_{2}$ & $-0,061$ & 0,160 & 0,099 \\
\hline & 19 & Total number of & Bolgar & $\mathrm{P}_{1}$ & $-0,078$ & 0,087 & 0,009 \\
\hline VI & & shoots & Ryssalka 1 & $\mathrm{P}_{2}$ & $-0,024$ & 0,284 & 0,260 \\
\hline & 20 & Total number of & Bolgar & $P_{1}$ & 0,048 & 0,009 & 0,057 \\
\hline & & fruiting shoots & Ryssalka 1 & $\mathrm{P}_{2}$ & 0,007 & 0,058 & 0,065 \\
\hline & 21 & Total number of & Bolgar & $\mathrm{P}_{1}$ & 0,013 & 0,014 & 0,027 \\
\hline & & clusters & Ryssalka 1 & $\mathrm{P}_{2}$ & 0,004 & 0,049 & 0,053 \\
\hline
\end{tabular}




\section{CONCLUDING REMARKS}

There are no highly significant traits for yield formation from the cultivar Bolgar, in which the correlation coefficients, direct and total indirect influences have positive values. With a total relative participation $95,6 \%$, the productivity of this cultivar is determined by traits, which are predominantly connected with the actual fertility of vines - total number of clusters (24,8\%) and fruiting shoots (17,9\%), fertility coefficients - totally $(16,5 \%)$.

Positive correlations have been established between the two parent cultivars and F1 progeny in the traits shoot and fruiting shoot fertility coefficient, cluster width, total number of shoots, fruiting shoots and clusters. For Bolgar they are higher regarding total number of buds, acids, budding-flowering and cluster length, and for Russalka 1 - main shoot fertility coefficient, millerandage berries and average cluster weight.

Positive direct influence determined by a moderate correlation, on the seedlings from $F_{1}$ progeny, is exerted by all studied fertility coefficients in the cultivar Bolgar. The correlations in the other traits and the influence of the separate parent cultivars have positive or negative value, which can be used in the selection of valuable elite forms.

\section{REFERENCES}

Bulgarian Ampelography (1990). General ampelography, Bulgarian Academy of Sciences. Agricultural Academy. Institute of Viticulture and Wine Production - Pleven. Sofia.

Golodriga, P.Y., Troshin, L.P., Frolova, L.I. (1985). Inheritance of the trait seedlessness in hybrid progeny in vine V. vinifera L.. Cytology and Genetics, 19, 372-376.

Lidanski, T, (1988). Statistical methods in biology and agriculture. Zemizdat, Sofia, 375.

Mokreva, T. (2004). Production function in analysis of yield structure in vine. Horticultural Sciences, 42, 130-137.

Mokreva, T. (2007). Comparative characteristics of statistical criteria and algorithms for evaluation of experimental data from viticulture. PhD Dissertation, Plovdiv, 145.

Pospisilova, D., Palenik, V. (1988). Heredity of seedlessness in grapes. Genetika a Slechteni, 24 /LXI/, 4, 325-332.

Rokitskii, P.F. (1973). Biological statistics. Third edition. Minsk, Higher Institute, 328.

Roychev, V. (2012). Ampelography. Academic Press of the Agricultural University Plovdiv, 574.

Smirnov, K.V. (1977). Vine selection for seedlessness. Moscow. Collection of scientific works, TSHA, 231, 90-97.

Troshin, L.P. (1990). Evaluation and choice of selection material in vine. Yalta, 136.

Troshin, L.P. (1997). Analysis of hereditary information in vine. Grape and Wine of Russia, 1,17-21.

Valchev, V. (1990). Intraspecific and interspecific hybridization in vine. Habilitation dissertation, Pleven, 272. 


\title{
ИСТРАЖУВАЊЕ НА КОРЕЛАЦИЈАТА ПОМЕЃУ КВАНТИТАТИВНИТЕ КАРАКТЕРИСТИКИ КОИШТО ГО ОДРЕДУВААТ ПРИНОСОТ КАЈ СОРТАТА БЛГАР И ХИБРИДОТ БЛГАР Х РУСАЛКА 1
}

\author{
Венелин Ројчев ${ }^{1 *}$, Душко Неделковски², Климе Белески ${ }^{2}$ \\ 'Земјоделски универзитет- Пловдив, Р. Бугарија \\ ${ }^{2}$ Универзитет Св.Кирил и Методиј - Скопје, Земјоделски институт- Скопје, \\ Република Северна Македонија \\ *Контактавтор: roytchev@yahoo.com
}

\begin{abstract}
Резиме
Корелациите помеѓу квантитативните карактеристики, коишто го одредуваат приносот кај сортата блгар и $\Phi_{1}$ потомок на хибридна комбинација блгар х русалка 1, беа испитани преку Раth анализа. Откриено е дека нема карактеристики коишто се многу значајни за формирање на принос од оваа сорта за кои коефициентите на корелација, директните и вкупните индиректни влијанија имаат позитивни вредности. Позитивни корелации се утврдени помеѓу двете родителски сорти и $\mathrm{F}_{1}$ потомството за својствата: развиени и родни ластари, коефициент на родност, ширина на гроздот, вкупен број на ластари, родни ластари и гроздови. Сите анализирани коефициенти на родност кај сортата блгар имаат позитивни директни влијанија, утврдени со умерени корелации, врз семениците од $F_{1}$ потомство. Корелациите на другите особини и влијанието на одделните родителски сорти имаат позитивни или негативни вредности кои можат да се користат при изборот на вредни елитни форми.
\end{abstract}

Клучни зборови: квантитативни карактеристики, родност, семена и бессемена сорта, F1, потомок 